\title{
Phase II Trial of Dendritic Cells Loaded with Antigens from Self-Renewing, Proliferating Autologous Tumor Cells as Patient-Specific Antitumor Vaccines in Patients with Metastatic Melanoma: Final Report
}

\author{
Robert O. Dillman, ${ }^{1}$ Senthamil R. Selvan, ${ }^{1}$ Patric M. Schiltz, ${ }^{1}$ Edward F. McClay, ${ }^{2}$ Neil M. Barth, ${ }^{1}$ \\ Carol DePriest, ${ }^{1}$ Cristina de Leon, ${ }^{1}$ Cheryl Mayorga, ${ }^{1}$ Andrew N. Cornforth, ${ }^{1}$ and Kanoe Allen ${ }^{1}$
}

\begin{abstract}
Between January 2001 and September 2007, we treated 54 metastatic melanoma patients with patient-specific tumor cell vaccines consisting of dendritic cells (DCS), derived from their peripheral blood cells that were cultured in interleukin (IL)-4 and granulocyte macrophage colony-stimulating factor (GM-CSF), which had phagocytosed irradiated autologous tumor cells from a continuously proliferating, self-renewing, autologus tumor cell (TC) culture. The loaded DCs were injected subcutaneously in $500 \mu \mathrm{g}$ of GM-CSF weekly $\mathrm{x}$ three, and then monthly for 5 months, for a total of up to 8 injections. The 34 men and 20 women had a median age of 50.5 years; 32 had M1c (visceral metastases and/or elevated lactate dehydrogenase) as their most advanced disease stage. Overall, $83 \%$ had received other systemic therapies, including interferon-alpha $(n=20)$, biochemotherapy $(n=19)$, GM-CSF $(n=19)$, chemotherapy $(n=16)$, IL-2 $(n=13)$, and other investigational vaccines $(n=7)$. Patients received an average of 7.4 vaccinations. Treatment was well-tolerated, with most patients experiencing only mild local pruritus and/or erythema. A positive delayed-type hypersensitivity reaction to purified autologous tumor cells was observed at baseline in only 1 of 54 patients, compared to 12 of 54 following vaccination $(p=0.001)$. The projected 5 -year survival rate is an impressive $54 \%$ at a median follow-up of 4.5 years (range, 2.4-7.4) for the 30 surviving patients. This survival was superior to that observed following vaccination with irradiated TC in 48 melanoma patients in a previous trial (64 versus 31 months, $p=0.016$ ). This patient-specific vaccine warrants further investigation, based on its safety and encouraging survival rates. (Clinical Trial Number: NCI-V01-1646).
\end{abstract}

Key words: metastatic melanoma, dendritic cells, tumor cell lines, vaccines, tumor stem cells

\section{Introduction}

$\mathbf{M}$ elanoma patients who experience recurrent disease in the form of regional metastases or distant metastases, or have distant metastatic disease at the time of diagnosis, are at high risk for additional metastases and death from melanoma. ${ }^{1}$ There is continued interest in developing new thera- pies for such patients, including antimelanoma vaccines, because there are no systemic therapies that can be relied upon to cure metastatic melanoma. ${ }^{1,2}$ One novel approach is to load autologous dendritic cells (DCs) with antigens from self-renewing, proliferating, autologous tumor cells (TCs).

Advantages for the use of proliferating, self-renewing, autologous TC as a source of antigen include: immunizing

\footnotetext{
${ }^{1}$ Hoag Cancer Center, Newport Beach, CA.

${ }^{2}$ Melanoma Research Center of San Diego, Vista, CA.

Presented, in part, at the 43rd Annual Meeting of the American Society for Clinical Oncology, Chicago, IL, June 3, 2007 (Abstract 8555).
}

Address correspondence to: Robert O. Dillman; Hoag Cancer Center; One Hoag Drive, Building 41, Newport Beach, CA 92658; Tel.: 949-764-8091; Fax: 949-764-8102

E-mail: rdillman@hoaghospital.org 
with antigens from the patient's own tumor, immunization with antigens from proliferating, self-renewing cells that, conceivably, represent tumor stem cells or progenitor cells, loading DC with antigens from pure tumor cells without contamination by normal cells, presentation of tumor antigens to DC in the context of the cell membrane on metabolically active cells, having a renewable source of tumor antigen, availability of pure tumor cells for patient-specific delayed-type hypersensitivity (DTH) testing, and a source of pure autologous tumor cells to test for tumor-specific immune responses in vitro. Such an approach is not feasible for patients with only a primary melanoma lesion and/or microscopic regional metastases, because all surgically removed tissue is consumed during the diagnostic evaluation by pathologists. However, regional recurrences and distant metastases often occur in sites that are readily accessible to surgical excision, which provides an opportunity to obtain fresh tissue from which to try to establish tumor cell lines.

We have investigated the safety and therapeutic potential of patient-specific autologous vaccines derived from short-term autologous tumor cell lines from various tumor types. $^{3-6}$ Most of this effort has involved patients with metastatic melanoma. We previously treated 74 metastatic melanoma patients with a tumor cell vaccine (TCV) consisting of irradiated tumor cells from their autologus tumor cell lines. ${ }^{7}$ After a median follow-up of 6 years, the median overall survival was 20.5 months with a $29 \%$ 5-year survival rate. In the current trial, we made three changes in an effort to improve the product, compared to that used in the previous trial. First, in the final steps of cell culture, we incubated proliferating TC with soluble recombinant interferon- $\gamma$ to increase the expression of human lymphocyte antigens (HLAs) I and II and, possibly, some tumor antigens. Second, we incubated the irradiated, cryopreserved TC with autologous DC generated from peripheral blood mononuclear cells (PBMCs), so that the final vaccine product was a dendritic cell vaccine $(\mathrm{DCV})$, rather than a TCV. Third, the final DCV product was suspended in granulocyte macrophage colony-stimulating factor (GM-CSF) for injection, rather than administering a concurrent injection of a cytokine adjuvant.

We previously reported the planned interim results for the first 20 patients enrolled in this trial, which showed that the DCV product was safe, that DTH reactions to autologous TC were being induced, and that initial survival results were encouraging. ${ }^{8}$ In a brief correspondence, we highlighted the long-term progression-free survival experienced by 6 patients after the completion of the DCV therapy. ${ }^{9}$ Other reports have highlighted some of the immune-monitoring and tumormarker studies carried out on subsets of patients enrolled in the current trial. ${ }^{10-13}$ The current report is the final clinical analysis of the phase II trial, carried out at a time when the median follow-up for surviving patients was 4.5 years.

\section{Methods}

The clinical protocol was approved by the Hoag Hospital Institutional Review Committee for the Protection of Human Subjects, the U.S. Food and Drug Administration (FDA; BBIND 8554), and reviewers for the National Cancer Institute's Physician Data Query (clinical trial number: NCI-V01-1646). All patients gave written informed consent prior to treatment.

\section{Patient population}

Patients were candidates for this protocol only if the Hoag Cancer Center Cell Biology Laboratory had been able to establish a continuously proliferating autologous TC line from which at least 200 million cells could be obtained. At the time of treatment, patients had to be 16 years of age or greater, with a diagnosis of regionally recurrent or distant metastatic melanoma, and meet usual phase II clinical trial criteria for hematologic, metabolic, renal, and hepatic chemistry tests, and have an Eastern Cooperative Oncology Group (ECOG) performance level of $0-2$. Patients with brain metastases were eligible if known tumors had been controlled with therapy and they were not taking pharmacologic doses of corticosteroids. Treatment was deferred until at least 1 month after any immunosuppressive therapy, including high-dose corticosteroids, chemotherapy, or radiation therapy. Patients were tested for reactivity to common recall antigens, but anergy was not an exclusion criterion. Presence of measurable disease at the time of treatment was not required for eligibility.

\section{Patient-specific DCV}

The DCV consisted of a combination of irradiated TC derived from a continuously proliferating autologous TC line, which was able to be expanded to 200 million cells and treated with $1000 \mathrm{U} / \mathrm{mL}$ interferon-gamma (IFN- $\gamma$ ) for 72 hours, and autologous DC. ${ }^{8}$ DCs were generated from PBMCs by preferential-monocyte adherence to plastic and culture in the presence of GM-CSF and interleukin (IL)-4. DCs and TCs were incubated overnight, during which time DCs phagocytosed TCs resulting in the final DCV product on which quality-control measures, including sterility testing, were conducted. Individual doses of vaccine were cryopreserved in liquid nitrogen in aliquots of about 10-20 million cells per dose.

\section{Treatment plan}

At the time of each treatment, one aliquot of cryopreserved cells was thawed and suspended in $500 \mu \mathrm{g}$ of GM-CSF for subcutaneous (s.c.) administration weekly for 3 weeks and then monthly for up to 5 additional months for a total of 6 months of therapy and up to 8 doses. At the discretion of their primary managing oncologists, patients were allowed to interrupt, and subsequently resume, therapy following the progression or appearance of new metastatic disease that could be eliminated by other treatment modalities.

\section{Clinical observations}

Toxicity was scored by using the NCI Common Terminology Criteria for Adverse Events. Tumor responses were defined according to Response Evaluation Criteria in Solid Tumors (RECIST) criteria for patients with objectively measurable disease (OMD). Patients with no measurable disease (NMD) were monitored for disease progression at a minimum of 3-month intervals by physical examination and photon emission tomography (PET) or computerized tomography (CT) scans and, if symptomatic, whole-body bone scan and brain magnetic resonance imaging (MRI). Because of uncertainties related to the sensitivity of the PET scan, 
compared to CT scans, and the uncertain significance of lessthan-1.0-cm lesions noted on CT scans, for some analyses, patients were categorized as having OMD, equivocal-nonmeasurable disease, and or evidence of disease.

\section{Statistical considerations}

Survival calculations were made from the date of the initial DTH test and from the date of completion of vaccine. Key end-point dates were date of death for overall survival (OS), and date of disease progression for progression-free survival (PFS). Means were compared by using the Student's $t$-test. Proportions were compared by the chi-square test or Fisher's exact test. Survival curves were compared by using the twotailed log-rank test.

\section{Measures of immune responses and tumor markers}

At baseline, and following completion of the first 3 weekly vaccinations, patients underwent intradermal (i.d.) tests for DTH to 1 million irradiated autologous tumor cells that had not been exposed to IFN- $\gamma$ and had been stored in serum-free medium. The area of induration was measured 48-72 hours after the injection. After the first few patients were enrolled, the protocol was amended to add a third tumor DTH test after 5 months of therapy, just prior to the last vaccine injection. Serum samples were obtained at the time of DTH testing for immune modulation assays that included the measurement of serum ganglioside and antiganglioside immunoglobulin levels, ${ }^{10}$ measurement of serum S100B and lactate dehydrogenase (LDH), ${ }^{11}$ measurement of serum cytokines by a qualitative cytokine array protein assay, ${ }^{12}$ secretion of IFN- $\gamma$ by autologous immune cells in response to stimulation by proliferating autologous tumor cells, ${ }^{12}$ and qualitative measurement of thymus activating-regulating cytokine (TARC) levels. ${ }^{13}$

\section{Results}

\section{Melanoma cell lines}

Between January 2000 and December 2006, the Hoag Cancer Center Cell Biology Laboratory received 227 metastatic melanoma specimens, from which efforts were made to establish cell lines for $196 .{ }^{14}$ Of these 227 samples, 31 were felt to be of inadequate quality for efforts to establish a cell line, 32 were discontinued early before success or failure could be ascertained, and 54 could not be grown. Initial success, defined by the expansion of a purified short-term tumor cell line to 50 million cells, was achieved for 110 of 227 $(48.5 \%)$ of all samples received, 110 of 196 (56.1\%) for all samples for which growth efforts were initiated, and 110 of $164(67.0 \%)$ for all good-quality samples that were not aborted early because of patient deterioration. The success rate was similar whether the tumor source was fresh $(82 / 122$; $67.2 \%)$ or cryopreserved specimens $(28 / 42 ; 66.7 \%)$. The length of time to a successful culture was similar for fresh (mean, $3.0 \pm 2.68$ months) and cryopreserved specimens (mean, $3.2 \pm 1.50$ months). The anatomic location of the tumor did not influence the probability of establishing a successful cell culture. It took an average of $3.1 \pm 2.5$ months (range, 0.4-14.7) to reach 50 million cells and an average of $4.5 \pm 2.4$ months to expand to 200 million cells, incubate with IFN- $\gamma$, and complete quality-control testing after irradiating tumor cells with $10 \mathrm{~Gy}$ via a cesium irradiator. A total of 96 lines were prepared for potential incubation with DC. For TC from the 54 patients who were treated, incubation with IFN- $\gamma$ was associated with an increase in HLA II positivity, from $48 \% \pm 40 \%$ to $80 \% \pm 26 \%(p<0.0001)$ and increase in median intensity of fluorescence (MIF) from $432 \pm 563$ to $704 \pm 687$. The increase in HLA1 was less impressive, from $92 \% \pm 15 \%$ to $97 \% \pm 5 \%(p=0.032)$ and MIF from $287 \pm 250$ to $760 \pm 466$.

\section{Dendritic cells}

DCs were produced successfully for all 67 patients who underwent leukopheresis to obtain PBMC. Overnight incubation was associated with phagocytosis of tumor cells and resulted in tumor-antigen loading based on immunohistochemical stains for melanoma-associated antigens and microscopic visualization (Fig. 1). DC cell numbers were similar before and after incubation with TC.

\section{$D C V$}

From the time of leukopheresis, it took approximately 4 weeks to prepare the DCV and complete quality-control testing. After incubation with TC, DC exhibited increased expression of CD80 and CD83 and decreased CD11, which is associated with DC maturation. DC from all 67 patients were incubated with TC to prepare a DCV product, but 11 patients were never enrolled in the trial because of clinical deterioration from progressive disease, 1 patient was never treated, and 1 was found to be ineligible.

\section{Clinical summary}

Fifty-six (56) patients with regionally recurrent or distant metastatic melanoma were enrolled; 55 patients received at least 1 dose of DCV, but 1 was ineligible because of concurrent treatment for metastatic prostate cancer, and 1 patient was withdrawn from the study by his managing physician prior to receiving a vaccine dose because of new brain and other distant metastases. Table 1 summarizes the characteristics of the

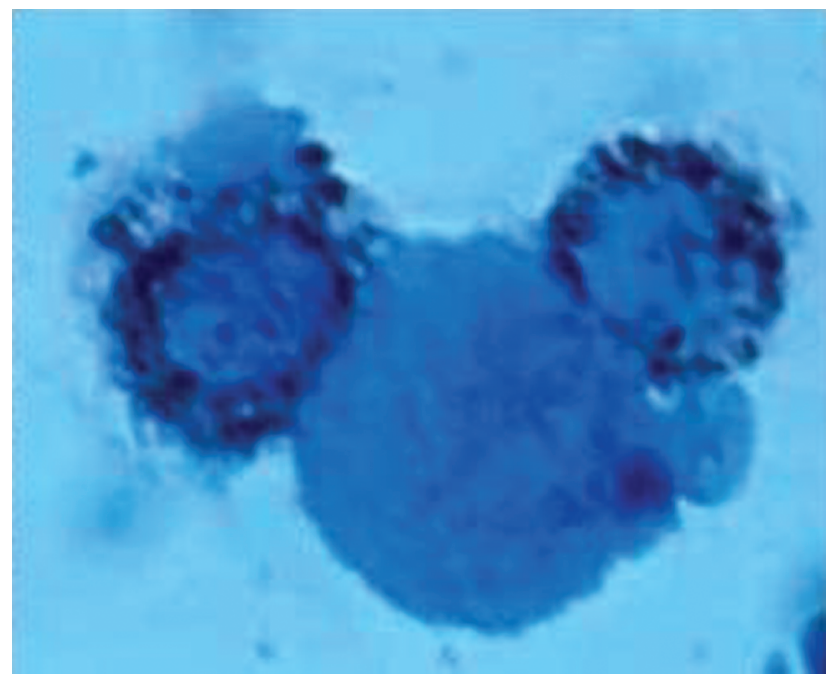

FIG. 1. Microscopic appearance of dendritic cell phagocytosing two melanoma cells whose granules are stained for HMB-45 melanoma antigens. 
Table 1. Patient and Tumor Characteristics

54 patients ( 30 men, 24 women)
median age, 50.5 years
Maximum extent of disease and sites of disease at start of
treatment
42 distant metastases
$\quad 15$ NMD
$\quad 17$ lung
$\quad 8$ soft tissue or cutaneous
$\quad 7$ lymph node
$\quad 5$ intra-abdominal
$\quad 4$ liver
$\quad 2$ bone
12 regional metastatic recurrences
$\quad 11$ NMD
$\quad 1$ soft tissue
Classification of metastatic melanoma at start of vaccine
treatment
26 no evidence of disease
15 measurable metastatic disease (OMD) \pm nonmeasurable
13 nonmeasurable disease (NMD)
Classification of metastatic melanoma by most advanced
stage since diagnosis
19 M1A Distant skin, s.c. or lymph node metastases
3 M1B Lung metastases only
32 M1C Visceral metastases or metastases plus increased
LDH (>537)
Prior local treatment for liver metastases
3 chemoembolization for liver metastases
1 surgery and radiofrequency ablation for liver metastases
State of residence
36 California
3 Colorado
2 Oregon
2 Nevada
2 Texas
2 Louisiana
1 Arizona
1 Wyoming
1 Nebraska
1 Oklahoma
1 New York
France

OMD, objectively measurable disease; LDH, lactate dehydrogenase.

54 eligible and evaluable patients; their prior treatments are summarized in Table 2. The 34 men and 20 women had a median age of 50.5 years; 32 had M1c (visceral metastases and/or elevated LDH) as their most advanced disease stage. Further, $83 \%$ had received other systemic therapies, including interferon-alpha $(n=20)$, biochemotherapy $(n=19)$, GM-CSF $(n=19)$, chemotherapy $(n=16)$, IL-2 $(n=13)$, and other investigational vaccines $(n=7)$.

The 54 patients received 400 vaccine injections for an average of 7.4 of a possible 8 injections. Forty-three (43) patients completed the entire series of vaccinations, but 14 did so only after an interruption for local treatment of progressive disease. Of the 11 patients who did not complete the planned treatment, 9 discontinued because of progressive disease, 1 was withdrawn because of noncompliance, and 1 died at home of an acute cerebral hemorrhage event after having received 3
Table 2. Prior Treatment

54 surgery

20 interferon-alpha alone

19 biochemotherapy combination with IL-2 and/or interferon

19 GM-CSF

16 chemotherapy alone

13 interleukin-2 alone

11 radiation therapy

7 vaccine

3 BCG

2 thalidomide

1 MDX-010 antibody

Prior chemotherapy agents

23 dacarbazine and/or temozolomide

20 cisplatin or carboplatin

8 nitrosourea

7 vinblastine or vinorelbine

Prior treatment for brain metastases

11 gamma knife radiosurgery for brain metastases

4 temozolomide

2 resection by craniotomy

IL-2, interleukin-2; GM-CSF, granulocyte macrophage colonystimulating factor; BCG, bacillus Calmette-Guerin.

injections. He did not have brain metastases, but was under treatment for hypertension and hypercholesterolemia. No patients have been lost to follow-up and all have been followed for more than 2 years since initiating the vaccine.

\section{Toxicity}

Overall, the vaccine was well tolerated, but every patient experienced symptoms that resulted in at least one grade 1 toxicity. Grade 1 injection-site reactions, such as mild erythema and pruritis, were experienced by $79 \%$ of patients. Several patients were given diphenhydramine and acetaminophen because of local reactions to the injections. Two (2) female patients reported hypermenorrhea during the first few weeks of treatment, which resolved. Of the 31 (57\%) grade 2 toxicities reported, 21 (39\%) were injection-site reactions. Two (2) patients experienced grade IV adverse events and 12 grade III, but only 2 of these (both grade III) were attributed to the vaccine. One (1) patient had rapid onset of grade III allergic symptoms after her last vaccine; she was treated with corticosteroids. Another patient experienced hives after her fifth vaccination; GM-CSF was withheld from her subsequent 3 vaccinations, which were well tolerated.

\section{Objective tumor response}

As summarized in Table 3, none of the 15 patients with OMD by RECIST criteria had an objective response. All have progressed with a median time to disease progression of 2.4 months. Six (6) patients had a best response of stable disease lasting 3.5, 4.1, 4.4, 7.1, 20.8, and 27.8 months from initiation of therapy, while 9 had progressive disease within 2.5 months of starting therapy.

\section{Tumor control}

The 39 patients who started the study with NMD had a median PFS of 24.7 months and a 39\% 5-year PFS. One (1) 
Table 3. Objective Responses, Tumor Control, and Survival by Tumor Burden

\begin{tabular}{|c|c|c|c|c|c|}
\hline Disease status & Response rate & $\begin{array}{c}\text { Progression } \\
\text { free }\end{array}$ & $\begin{array}{l}\text { Still } \\
\text { alive }\end{array}$ & $\begin{array}{l}\text { Median } \\
\text { survival } \\
\text { (months) }\end{array}$ & $\begin{array}{c}5-y e a r \\
\text { survival (\%) }\end{array}$ \\
\hline Measurable & Objective (RECIST) 0/15 & $0 / 15$ & $5 / 15$ & 17.7 & 27 \\
\hline Nonmeasurable & Evaluable 0/13 & $3 / 13$ & $7 / 13$ & 53.7 & 46 \\
\hline No evidence & $\mathrm{N} / \mathrm{A}$ & $9 / 26$ & $18 / 26$ & 61.0 & 57 \\
\hline
\end{tabular}

Median follow-up; 4.5 years for survivors (range, 2.2-7.4).

RECIST, response evaluation criteria in solid tumors; N/A, not applicable; NR, not reached.

NMD patient died at 1.3 months of a cerebral hemorrhage that was unrelated to therapy or melanoma. Twelve (12) patients classified as NMD at the time of treatment have neither progressed nor died after follow-up from 34 to 86 months.

\section{Survival}

Figure 2 shows the overall survival (OS) from the start of treatment. Most disease progression occurred within the first 12 months. There have been 24 deaths, 23 from progressive metastatic melanoma and 1 a non-melanoma-related cerebrovascular event. The median survival for all 54 eligible and evaluable patients has not been reached at a median followup of 54 months for the 30 survivors (range, 29-89). The projected 5 -year survival rate is $54 \%$. Successive survival rates at $1,2,3,4$, and 5 years are $85 \%, 72 \%, 64 \%, 57 \%$, and $54 \%$ after initiation of vaccine therapy. Median PFS is 4.2 months and 5 -year PFS is $23 \%$, with 12 patients alive without disease progression, all more than 3 years since initiating vaccine.

The total cohort of $15 \mathrm{OMD}$ patients had an inferior survival, compared to the other 39 patients (5-year survival $68 \%$ versus $27 \%$; $p=0.006$ ). As shown in Figure 3, there was no difference in survival for the subset who had no evidence of disease (NED; $n=26$ ), compared to $13 \mathrm{NMD}$ patients who had equivocal, or detectable, but nonmeasurable disease. The 22 patients with M1a or M1b disease had a better survival than the 32 patients with M1c disease (5-year survival $81 \%$ versus $41 \% ; p=0.008)$. There was no difference in survival by gender, by whether patients had previously received chemotherapy, by whether they had received prior bio-

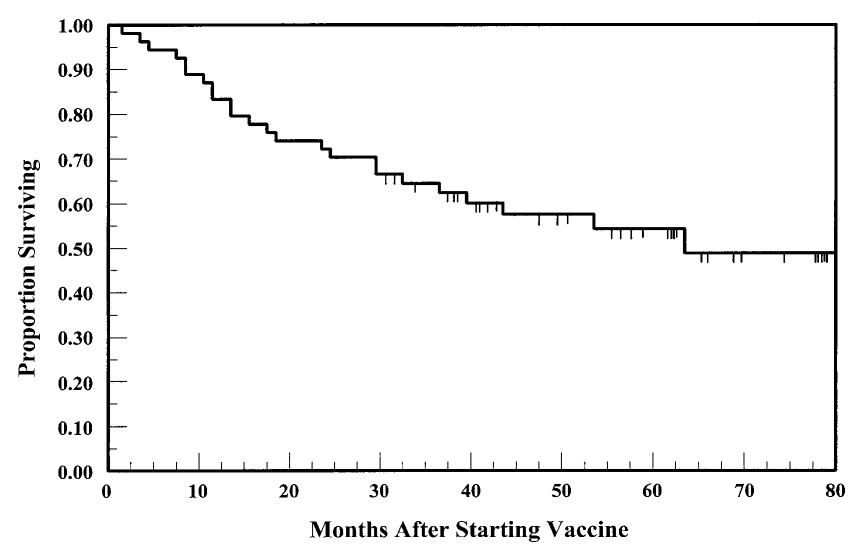

FIG. 2. Survival curves for all 54 patients. Median overall survival (OS) not reached with median follow-up of 54 months for 32 survivors and 5-year OS $=54 \%$. chemotherapy, or by whether their residence was in California. However, the 27 patients 50 years of age or younger had a better survival than the 27 older patients (5-year survival $83 \%$ versus $32 \%$; $p=0.002$ ).

Figure 4 compares the survival of the 54 patients treated in this trial, to a historical cohort of 48 patients who received at least 3 weekly vaccinations in a previous trial in which patients were injected with irradiated tumor cells (TCV) and various adjuvants. ${ }^{7}$ The characteristics of the two patient populations are summarized in Table 4. Major differences were in the proportion of patients who received GM-CSF $(p<0.0001)$ and the proportion who were treated at Hoag Cancer Center $(p<0.0001)$. Median survival (64 versus 31 months) and 5-year survival (54\% versus $31 \%$ ) were better for the patients who received the DCV $(p=0.016)$.

\section{Case reports}

The 8 patients summarized below are remarkable for the long-term PFS experienced after completing the vaccine, even though they had widely metastatic disease and/or repeated appearance of new metastases despite various therapies. Some of these were included in less detail in previous reports. 8

Patient 1. A 58-year-old male was diagnosed with a deepscalp melanoma in June 1999. In March 2000, he had a left neck recurrence that was resected and a second regional recurrence in May 2000, from which a TC line was established.

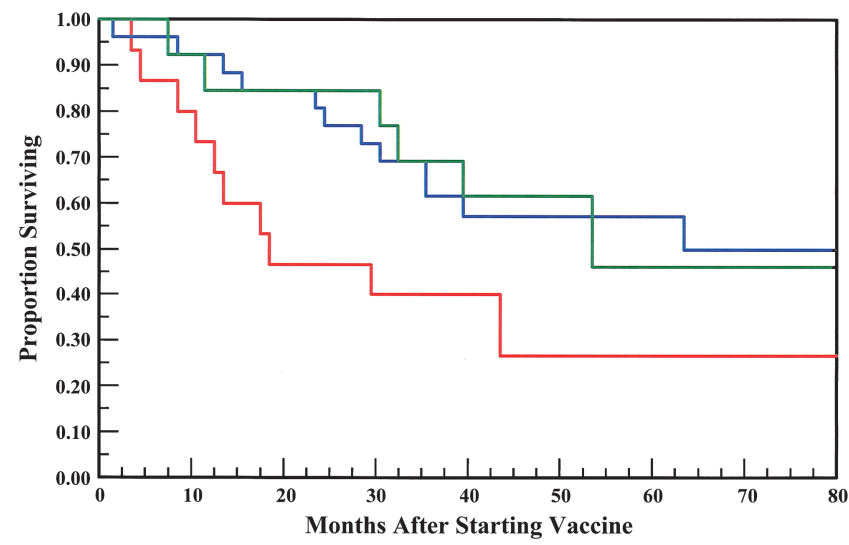

FIG. 3. Survival curves by tumor burden at the time vaccine therapy was initiated. NED (blue), no evident disease $(n=26)$; NMD (green), nonmeasurable disease $(n=13)$; OMD (orange), objectively measurable disease $(n=15)$. The combination of NED and NMD $(n=39)$ was superior to OMD $(p=0.026)$. 


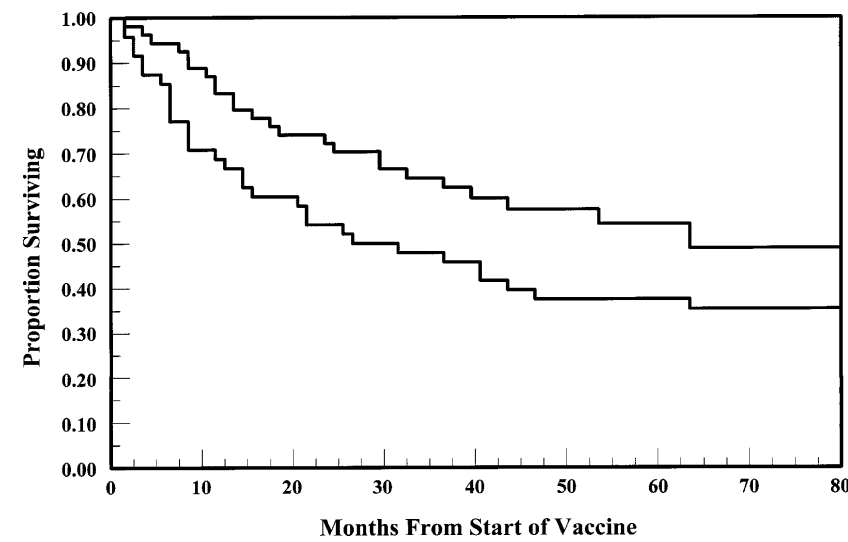

FIG. 4. Superior survival for metastatic melanoma patients receiving dendritic cell vaccine $(n=54 ; 2001-2007)$ versus tumor cell vaccine $(n=48,1992-2000)$ (64 versus 31 months, 5 -year survival $54 \%$ versus $38 \%$; $p=0.043$ ).

In July 2000, he was treated with cisplatin, IL-2, IFN- $\alpha$, and tamoxifen. A DCV was started in December 2000, but a left neck metastasis increased in size and was resected in February 2001. The DCV was completed in June 2001. A local recurrence near the original scalp lesion was resected in February 2003, 20 months after completing the vaccine. Additional chemotherapy was given at that time. He has remained disease free since then, more than 7 years after completing the vaccine, and more than 5 years since the last lesion of any type was resected.

Patient 2. A 45-year-old female attorney presented with lung and brain melanoma metastases in February 2000. The lung lesion was resected and the brain lesion was treated by gamma knife. From March to June 2000, she received an inpatient hybrid bolus and continuous infusion IL-2 regimen. In July 2000, she had new adrenal and brain metastases. The brain lesion was treated with a gamma knife and she began

Table 4. Characteristics of Patients in Successive Trials of Tumor Cell Vaccine

\begin{tabular}{lcc}
\hline Variable & $\begin{array}{c}\text { Tumor cell } \\
\text { vaccine } \\
(\mathrm{N}=48)\end{array}$ & $\begin{array}{c}\text { Dendritic cell } \\
\text { vaccine } \\
(\mathrm{N}=54)\end{array}$ \\
\hline Median age in years & 46 & 50.5 \\
\% female & 48 & 37 \\
\% NED at time of therapy & 54 & 48 \\
\% Anergic at baseline & 23 & 23 \\
Mean injections & 6.7 & 7.4 \\
\% visceral metastases & 31 & 25 \\
\% GM-CSF & 48 & 100 \\
\% treated at Hoag & 50 & 100 \\
Number dead & 33 & 24 \\
Med f/u of living & 86.3 months & 54.1 months \\
Median survival & 31 months & 64 months \\
2-year survival & $54 \%$ & $72 \%$ \\
5-year survival & $37 \%$ & $54 \%$ \\
\hline
\end{tabular}

$(n=48,1992-2000)$ and dendritic cell vaccine $(n=54,2001-2007)$.

${ }^{a}$ Brain, bone, liver, or lung.

NED, no evidence of disease; GM-CSF, granulocyte macrophage colony-stimulating factor. oral temozolomide. In September 2000, treatment was changed to bis-chloronitrosourea (BCNU), cisplatin, dacarbazine, and tamoxifen because of progression of the adrenal lesion. Because of further progression, this lesion was resected in February 2001, from which a cell line was established. When she started the DCV in May 2001, there was suspicion of recurrence of tumor in the right adrenal bed, which doubled in size during the first 4 vaccinations. Biopsy confirmed melanoma; so, the adrenal bed was treated with radiation therapy. She resumed the vaccine in September 2001 and completed therapy in December 2001. She remained in continuous remission for nearly 6 years. In June 2005, she was found to have right axillary adenopathy, which led to an axillary dissection with the finding that none of the 0 of 16 nodes were positive for cancer, with the largest being a 1.8-cm hyperplastic node. However, in November 2007, her husband noted a small lesion on her left lower back, and a punch biopsy of a subcutaneous nodule revealed melanoma, S100, and melan-A positive. Since then, she has had several other cutaneous nodules removed, but none of these were melanoma. At the time of this report, she was still alive and disease free. In contrast to the 6-year PFS experienced after completing the DCV, during the year after diagnosis before starting the vaccine, her longest progression-free interval had been only 4 months.

Patient 3. A 43-year-old female baker's assistant was diagnosed in March 1998 with melanoma of the right leg. A sentinel node biopsy was positive for micrometastatic disease; there was one additional malignant node discovered during lymphadenectomy. One (1) month after completing a year of high-dose adjuvant IFN- $\alpha$, she developed cutaneous nodules on the right leg. From June 1999 to January 2000, she received an investigational peptide vaccine, but in March 2000, a buttock recurrence was resected. A recurrence in May 2000 led to an additional inguinal node resection. During September and October 2000, she received cisplatin, IL-2, IFN- $\alpha$, and tamoxifen. In November 2000, she underwent the resection of a pelvic recurrence, from which a cell line was established. In March 2001, she underwent a more extensive pelvic node resection. Vaccine treatment was started in May 2001 and completed in August 2001. She has remained disease free more than 7 years after completing the vaccine. In contrast, during the first 3 years after her original diagnosis, her longest progression-free interval had been the initial 13 months.

Patient 4. A 16-year-old female high school student was diagnosed with a left leg melanoma in June 1999. A positive sentinel node and two additional malignant nodes were found in August 1999 during inguinal and iliac lymphadenectomy. From October to December 1999 she received 4 cycles of adjuvant cisplatin, IL-2, IFN- $\alpha$, and tamoxifen. In November 2000, a left pelvic recurrence was resected, from which a cell line was established. She received GM-CSF injections from January to April 2001, then started the DCV in June 2001 and completed therapy in November 2001. She graduated from college in June 2006, married, and has entered nursing school. She remains free of metastatic disease more than 7 years after completing the vaccine. In contrast, previously, her longest progression-free interval had been 15 months after the initial diagnosis. 
Patient 5. A 50-year-old physician presented in July 2001 with right chest pain that led to the finding of a lytic metastasis in a right rib. Further evaluation revealed extensive metastatic disease, including a $6.5-\mathrm{cm}$ pelvic mass, two lung metastases (largest, $3.0 \mathrm{~cm}$ ), mediastinal adenopathy, and diffuse bone metastases in the spine, right clavicle, and left scapula. A cell line was established from the resected pelvic lesion. From August to December 2001, he was treated with cisplatin, vinblastine, DTIC, IL- 2 , and IFN- $\alpha$, resulting in a near-complete remission. He received the vaccine from February to June 2002, and despite the widespread metastatic disease, he has remained disease free more than 6 years after completing the vaccine therapy.

Patient 6. A 51-year-old male was diagnosed with what was originally interpreted as an in situ melanoma in the left preauricular region in March 2000. However, a palpable mass led to a left superficial protidectomy on August 21, 2001, with 1 of 35 nodes positive for metastatic melanoma. He was given radiation therapy to the left neck. Additional tests identified a melanoma metastasis that was resected 1 month later; an autologous TC line was established from this metastasis. From May to November 2002, he completed the planned course of DCV therapy; then, in December 2002, he underwent the resection of a $6.0-\mathrm{cm}$ celiac node metastasis that had been noted in October 2002 and was suspected to have been growing throughout treatment. He has remained disease free nearly 6 years since the last surgical resection. In contrast, his previous longest disease-free interval had been 17 months after the original diagnosis of in situ melanoma.

Patient 14. A 20-year-old female college student presented in July 2002 with a melanoma on the back and bulky left axillary adenopathy with 12 of 19 nodes positive for metastatic disease. By September 2002, she had already experienced a recurrence in the left axilla and the chest wall, from which a cell line was established. From September to December, she received BCNU, cisplatin, dacarabazine, IL-2, and IFN- $\alpha$. In June 2003, she underwent enucleation of the right eye because of metastasis, and began the DCV 1 month later. In August 2003, she had gamma-knife treatment of a solitary brain metastasis and completed the DCV in November 2003. She remained disease free for 2.5 years until May 2006, when she developed additional brain metastases, but she has not had additional metastases outside the central nervous system. In contrast, previously, her longest interval of PFS had been 9 months. Additional brain metastases have been treated by gamma knife, temozolomide, and bevacizumab. As of November 2008, she was still alive 5 years after completing the vaccine.

Patient 22. A 56-year-old attorney had an incidental finding of a single melanoma lung metastasis that was resected in March 2002. From April 2002 to April 2003, he received bacillus Calmette-Guerin (BCG) and a placebo for an allogeneic melanoma cell line as part of a randomized, clinical trial. In May 2003, he underwent resection of a small bowel metastasis. From June to September 2003, he was retreated with the BCG-placebo, which was discontinued because of a liver metastasis that was resected and treated with radiofrequency ablation in November 2003. From December 2003 to June 2004, he received the DCV vaccine and remains disease free as of November 2008, nearly 4.5 years since completing the vaccine. In contrast, previously, his longest progression-free interval had been 1 year.

\section{Immune response and tumor markers}

Tumor markers. As previously reported, sustained low levels of serum ganglioside levels and/or a postvaccination decline in serum ganglioside levels were associated with better survival among 34 patients for whom such data were available. ${ }^{9}$ Both lactate dehydrogenase (LDH) and S100b were associated with tumor burden, but S100b was a better predictor of survival than LDH for the 45 patients for whom S100b measurements were available. ${ }^{10}$ An increase in S100b between baseline and week 4 after 3 vaccinations was predictive of a worse survival. An increase in TARC at week 4 was associated with improved survival. ${ }^{13}$

B-cell immunity. As previously reported, high levels of antiganglioside antibodies, and/or an increase in antiganglioside antibodies, were associated with better survival in the subset of patients for whom samples were available. ${ }^{9}$

T-cell immunity. At baseline 12 of 54 (22\%) were anergic to skin tests for standard recall antigens. Only 1 of 54 patients had a positive DTH test at baseline, compared to 12 of 54 who had a positive DTH at week 4 and/or week 24 $(p=0.003)$. DTH tests were positive at one or more of the three time points for $20 \%$ of the 15 OMD patients, compared to $26 \%$ of the $39 \mathrm{NMD}$ patients $(p=0.43)$. DTH positivity was not associated with superior survival $(p=0.363)$. A tumor-cell-specific enzyme-linked immunosorbent spot (ELISPOT) assay detected an increase in inducible IFN- $\gamma$ at week 4 in 15 of 39 (38\%) of patients, but this was not predictive of improved survival. ${ }^{12}$

Cytokines. Of the 20 patients evaluated for serum levels of 40 different cytokines in the cytoarray assay, after the first 3 vaccinations, there was a significant increase only in TARC, but decreases were noted in the levels of MIP-1, RANTES (regulated upon activation, normal T-cell expressed and secreted), angiogenin, oncostatin-M, vascular endothelial growth factor, and leptin. ${ }^{12}$ In a subsequent analysis, increases in TARC at week 4 were predictive of better survival. ${ }^{13}$

\section{Discussion}

This trial shows that patient-specific vaccines derived from autologous, self-renewing, proliferating melanoma cells and autologous DC (1) can be produced for a high percentage of patients, (2) are well tolerated when administered s.c. with GM-CSF, and (3) are associated with an encouraging 5 -year survival rate in patients with metastatic melanoma whose disease has been minimized by other therapies. Although no objective tumor regressions were seen in 15 patients who had OMD, prolonged PFS was observed from completion of the vaccine, which has led to speculation that the vaccine might be inducing an immune response against tumor stem cells or early progenitor cells. ${ }^{9}$ Since the antigen source in this trial was from self-renewing cells cultured in vitro, it is possible that terminally differentiated melanoma cells in measurable tumor in vivo may not contain the most important antigens for a response to the cells responsible for 
perpetuation of recurrent tumor despite other therapies. This may explain the surprising postvaccine PFS exceeding more than 3.5 years in several patients who previously had not had a PFS longer than a few months. ${ }^{9}$ Most of those patients have continued in remission for more than 5 years. This may also explain why vaccines directed against antigens that are expressed on differentiated melanoma cells have not demonstrated survival benefit. ${ }^{17-23}$ Although tumor cell vaccines derived from allogeneic cell lines offer many of the theoretic advantages as the autologous cell lines, they may lack critical antigens for specific patients, and this may explain the apparent lack of benefit for such vaccines in randomized trials. $^{16,24-28}$

The 1- and 5-year survival rates of $85 \%$ and $54 \%$, respectively, are remarkable for melanoma patients with documented metastatic disease. A meta-analysis of phase II cooperative group trials in stage IV melanoma recognized a 1 -year OS of $25 \%$, with 1 -year survival rates as high as $40 \%{ }^{15}$ However, patients enrolled in those trials typically had to have measurable disease as an entry criteria for therapy in a phase II trial. We previously observed a 5-year survival rate of $29 \%$ for metastatic melanoma patients who were treated with patient-specific vaccines consisting of irradiated TC and, most often, adjuvant GM-CSF and/or IFN- $\gamma \cdot{ }^{7}$ A 5-year survival rate of $40 \%$ was recently reported for patients rendered free of metastatic disease by surgical resection alone, followed by treatment with BCG plus placebo or BCG plus a two-cell allogeneic tumor cell vaccine. ${ }^{16}$ The 26 patients in our trial who had no evidence of cancer at the time vaccine therapy was initiated have a 5-year survival rate of $74 \%$.

The patients described in the case reports are remarkable for the long periods of disease control that were documented after completion of vaccine therapy. A vaccine directed against tumor stem cells or early self-renewing, proliferating cells would not be expected to produce antitumor effects against gross tumors, which mostly consist of more differentiated tumor cells that may lack antigens that are only present on the tumor stem cells. The failure to produce tumor responses in patients with measurable disease, and the growth of some tumors during vaccination, but followed by subsequent long-term PFS, supports the possibility that the vaccine is inducing an immune response against tumor stem cells or tumor progenitor cells that have prevented emergence of other possible sites of tumor.

There are several challenges to this approach, especially in comparison to a traditional product paradigm. These include: (1) a new product has to be developed for each individual patient; (2) a cell line cannot be successfully established for every patient; (3) it typically takes several months to establish a cell line to create the therapeutic product; (4) it appears that other therapies must be effective in reducing tumor burden for there to be a benefit from the $\mathrm{TCV}$. During the years this trial was being conducted, many patients could not be treated because a cell line could not be established. What is more frustrating is that many patients for whom a cell line could be established also were never treated because other therapies could not produce a state of limited disease. In addition to the 55 patients who actually received their DCV product, partial products were prepared for another 38 patients who were never treated. This included 13 patients, for whom we had prepared a final DCV, and an additional 25 patients on whose behalf tumor cell lines were incubated with gamma-interferon, but the tumor cell product was never combined with DCs. In all of these instances, patients had progressive disease, which either led to decisions by their managing physicians to pursue other therapies and/or deterioration in performance status such that patients were no longer eligible for treatment.

In 2006, this product was granted an orphan drug designation because of the limited melanoma population who might benefit from such patient-specific autologous tumor cell line-derived product. In 2007, we opened a randomized phase II trial to compare the two different vaccines we have produced and tested in clinical trials. One product consists of irradiated autologous tumor cells from short-term cell lines (BB-IND 5838), and the other consists of DCs loaded with material from irradiated autologous tumor cells from shortterm cell lines (BB-IND 8554).

\section{Acknowledgments}

The authors would like to recognize the laboratory contributions of Andrea Beatty, BS, Jared Novak, BS, Sarah Hendry, BS, and Karen Spencer, BS, CLS, and the clinical participation of James Jakowatz MD, Anton Bilchik, MD, Robert Weber, MD, Khosrow Mahdavi, MD, and Louis A. VanderMolen, MD. This work was supported by the Hoag Hospital Foundation.

\section{Disclosure Statement}

No competing financial conflicts exist.

\section{References}

1. Balch CM, Soong SJ, Gershenwald JE, et al. Prognostic factors analysis of 17,600 melanoma patients: Validation of the American Joint Committee on Cancer melanoma staging system. J Clin Oncol 2001;19:3622.

2. Tsao H, Atkins MB, Sober AJ. Management of cutaneous melanoma. NEJM 2004;351:998.

3. Dillman RO, Nayak SK, Beutel L. Establishing in vitro cultures of autologous tumor cells for use in active specific immunotherapy. J Immunother 1993;14:65.

4. Dillman RO, Beutel LD, Barth NM, et al. Irradiated cells from autologous tumor cell lines as patient-specific vaccine therapy in 125 patients with metastatic cancer: Induction of delayed-type hypersensitivity to autologous tumor is associated with improved survival. Cancer Biother Radiopharm 2002;17:51.

5. Dillman RO, Barth NM, VanderMolen LA, et al. Autologous tumor cell line-derived vaccine for patient-specific treatment of advanced renal cell carcinoma. Cancer Biother Radiopharm 2004;19:570.

6. Dillman RO, Beutel LD, DePriest C, et al. Cancer vaccine potency: Is there a dose/response relationship for patientspecific vaccines and clinical outcomes? Cancer Biother Radiopharm 2005;20:373.

7. Dillman RO, DePriest C, DeLeon C, et al. Patient-specific vaccines derived from autologous tumor cell lines as active specific immunotherapy: Results of exploratory phase I/II trials in patients with metastatic melanoma. Cancer Biother Radiopharm 2007;22:309.

8. Dillman RO, Selvan SR, Schlitz PM, et al. Phase I/II trial of melanoma patient-specific vaccine of proliferating autologous tumor cells, dendritic cells, and GM-CSF: Planned interim analysis. Cancer Biother Radiopharm 2004;19:658. 
9. Dillman RO, Selvan SR, Schlitz PM. Patient-specific dendritic cell vaccines for metastatic melanoma. NEJM 2006; 355:1179.

10. Selvan SR, Dillman RO, Fowler AW, et al. Monitoring response to treatment in melanoma patients: Potential of a serum glycomic marker. Internat J Cancer 2008;122:1374.

11. Schiltz PM, Dillman RO, Korse CM, et al. Lack of elevation of serum S100B in patients with metastatic melanoma as a predictor of outcome after induction with an autologous vaccine of proliferating tumor cells and dendritic cells. Cancer Biother Radiopharm 2008;23:214.

12. Cornforth A, Selvan RS, Fowler A, et al. Immune monitoring during the course of adaptive immunotherapy using autologous dendritic cells loaded with gamma-interferon treated, irradiated autologous tumor cells in patients with metastatic melanoma. Proc Am Assoc Cancer Res 2008;49:678. [Abstract 2865]

13. Cornforth AN, Lee GJ, Fowler AW, et al. Autologous dendritic cell immunotherapy-induced increases in serum tarc/ccl17 levels are associated with progression-free survival in advanced melanoma patients. International Society for Biological Therapy of Cancer 23rd Annual Meeting, San Diego, CA, October 30-November 2, 2008. J Immunother 2008;31:935.

14. Selvan SR, Carbonell DJ, Fowler AW, et al. Establishment of melanoma cell lines for autologus vaccine therapy: Comprehensive analysis of seven-year feasibility. International Society for Biological Therapy of Cancer 23rd Annual Meeting, San Diego, CA. November 1, 2008. J Immunother 2008;31:949.

15. Korn EL, Liu P-Y, Lee SJ, et al. Meta-analysis of phase II cooperative group trials in metastatic stage IV melanoma to determine progression-free and overall survival benchmarks for future phase II trials. J Clin Oncol 2008;26:527.

16. Morton DL, Mozzillo N, Thompson JF, et al. An international, randomized, phase III trial of bacillus Calmette-Guerin (BCG) plus allogeneic melanoma vaccine $(\mathrm{MCV})$ or placebo after complete resection of melanoma metastatic to regional or distant sites. J Clin Oncol 2007;25:474s. [Abstract 8508]

17. Sondak VK, Liu P-Y, Tuthill RJ, et al. Adjuvant immunotherapy of resected, intermediate-thickness, node-negative melanoma with an allogeneic tumor vaccine: Overall results of a randomized trial of the Southwest Oncology Group. J Clin Oncol 2002;20:2058.
18. Phan GQ, Touloukian CE, Yang JC, et al. Immunization of patients with metastatic melanoma using both class I- and class II-restricted peptides from melanoma-associated antigens. J Immunother 2003;26:349.

19. Chapman PB, Williams L, Salibi N, et al. A phase II trial comparing five dose levels of BEC2 anti-idiotypic monoclonal antibody vaccine that mimics GD3 ganglioside. Vaccine 2004;22:2904.

20. Berd D. M-Vax: An autologous, hapten-modified vaccine for human cancer. Expert Rev Vaccines 2004;3:521.

21. Slingluff CL, Jr, Petroni GR, Yamshchikov GV, et al. Clinical and immunologic results of a randomized, phase II trial of vaccination using four melanoma peptides either administered in granulocyte-macrophage colony-stimulating factor in adjuvant or pulsed on dendritic cells. J Clin Oncol 2003;21:4016.

22. Tagawa ST, Cheung E, Banta W, et al. Survival analysis after resection of metastatic disease followed by peptide vaccines in patients with stage IV melanoma. Cancer 2006;106:1353.

23. Testori A, Richards J, Whitman E, et al. Phase III comparison of vitespen, an autologous tumor-derived heat shock protein gp96 peptide complex vaccine, with physician's choice of treatment for stage IV melanoma: The C-100-21 Study Group. J Clin Oncol 2008;26:955.

24. Wallack MK, Sivanandham M, Balch CM, et al. Surgical adjuvant active specific immunotherapy for patients with stage III melanoma: The final analysis of data from a phase III, randomized, double-blind, multicenter vaccinia melanoma oncolysate trial. J Am Coll Surg 1998;187:69.

25. Bystryn JC, Zeleniuch-Jacquotte A, Oratz R, et al. Doubleblind trial of a polyvalent, shed-antigen, melanoma vaccine. Clin Cancer Res 2001;7:1882.

26. Hersey P, Coates AS, McCarthy, et al. Adjuvant immunotherapy of patients with high-risk melanoma using vaccinia viral lysates of melanoma: Results of a randomized trial. J Clin Oncol 2002;20:4181.

27. Hsueh EC, Morton DL. Antigen-based immunotherapy of melanoma: Canvaxin therapeutic polyvalent cancer vaccine. Semin Cancer Biol 2003;13:401.

28. Mitchell MS, Abrams J, Thompson JA, et al. Randomized trial of an allogeneic melanoma lysate vaccine with low-dose interferon alfa- $2 \mathrm{~b}$ compared with high-dose interferon Alfa$2 \mathrm{~b}$ for resected stage III cutaneous melanoma. J Clin Oncol 2007;25:2078. 
